\title{
The early years of life. Are they influenced by our microbiome?
}

\author{
Adam Jagodzinski ${ }^{1}$, Ewa Zielinska ${ }^{1}$, Lukasz Laczmanski ${ }^{2}$, Lidia Hirnle ${ }^{1}$ \\ ${ }^{1} 1$ st Department and Clinic of Gynaecology and Obstetrics, Wroclaw, Poland \\ ${ }^{2}$ Ludwik Hirszfeld Institute of Immunology and Experimental Therapy Polish Academy of Sciences, Wroclaw, Poland
}

\begin{abstract}
Human microbiome contains the genetic pool of bacteria and other microbes such as Achaea, fungi and viruses inhabiting the human body. It holds an immense potential to affect both physiological and pathological processes. The microbiome's composition can be defined in detail by analyzing ribosomal 16S rRNA and metagenomic tests. Recent increases in cesarean sections, the use of antibiotics during pregnancy, the increasing amount of prematurely born children and changes in infant nutrition have an impact on the microbiome forming process. A correlation between the bowel microbiome's composition and the occurrence of certain diseases, especially inflammatory bowel diseases (IBD), asthma and type 1 diabetes has been demonstrated. The influence on the development of cognitive functions and behaviour has also been displayed. This correlation justifies attempts to restore the beneficial the composition of the microbiome through the use of probiotics, vaginal microflora transfer in case of cesarean section and encouraging breastfeeding. Development of multiple studies on the topic of the human microbiome and its impact on the human body is necessary in order to reach final conclusions. The aim of this article is to summarize recent findings regarding the development of the human microbiome from the first days of life and the influence of changes in its composition on human health.
\end{abstract}

Key words: microbiome; newborn; delivery; diet; neurodevelopment; atopy; type 1 diabetes

Ginekologia Polska 2019; 90, 4: 228-232

\section{INTRODUCTION}

The human body is temporarily or permanently inhabited by abundant microbes, whose number varies from equal to triple the amount of human cells, depending on the source material. They are not distributed randomly; instead, they colonize certain areas, such as skin, upper respiratory tract, digestive tract and reproductive tract. The progress in the fields of microbiology, immunology and the expansion of new microbe identification methods have led to increased interest in the human microbiome. This term refers to the genetic pool of bacteria, Archaea, fungi, Protist and viruses inhabiting the human body. Regardless of the differences in the microbiome composition between specific individuals, distinct body areas contain similar microbe species. Microbiome's influence on the functioning of the human body is an important and fascinating aspect of the research papers. Its effect on the immune, endocrine and nervous systems has been featured in multiple publications [1]. Numerous prenatal and postnatal factors, such as the delivery method, intrauterine environment, perinatal antibiotic therapy, the nature and order of introduced food, genetic and environmental factors cause changes in the microbiome. Our intent is to review the current knowledge on the microbiome development in subsequent stages of human life, with particular emphasis on the first three years; and its impact on the functioning of the body.

\section{Microbiome identification}

There are many methods for microbiome analysis:

- Culturing of bacteria - the gold standard for microbial identification/not all species can be cultured

- 16S rRNA sequence analysis by Sanger sequencing - it allows identifying only one bacteria species in one test. It is impossible to sequence different $16 \mathrm{~s}$ rRNA in one test [2].

- $\mathrm{qPCR}$ - it allows to identify and quantify one bacteria species in one test.

- MALDI-TOF MS (matrix-assisted laser desorption/ionization time-of-flight mass spectrometry) - a new reliable tool for identification of bacteria and fungi cultivated on agar plates or in liquid media [3]. 
Therefore the most efficient method is a high-throughput sequencing based on NGS technique. This method allows profiling whole microbial community by sequencing of $16 \mathrm{~s}$ rRNA fragment gene. This analysis is possible because a small fragment of the 16s rRNA gene (ex. V3 and V4 regions) sequence is unique for many community analyses, including those based on a phylogenetic tree. This analytic process is divided into four main stages: DNA isolation, amplification of the 16s rRNA gene fragment, NGS analysis, clustering using Operational Taxonomic Unit method and annotation (bacterial' type identification). This workflow gives us information about species and the amount of bacteria present in our sample. MALDI-TOF MS allows identification of micro-organisms direct from positive blood cultures, subtyping, and drug susceptibility detection.

\section{MICROBIOME'S IMPACT ON STAGES OF HUMAN'S DEVELOPMENT}

\section{Prenatal period}

For many years, the intrauterine environment has been considered to be sterile during an uncomplicated pregnancy. Currently, however, this view is getting questioned by certain researchers [4]. Research on amniotic fluid, umbilical cord blood, fetal membranes, placenta and meconium from pregnancies delivered in the term, both through a vaginal birth and the cesarean section has shown a presence of microbes in the samples [5, 6]. One hypothesis implies a vertical bacteria transfer from the maternal microbiota. It is supported by the results of a study performed on mice, which has shown the presence of genetically marked $\mathrm{E}$. faecium in the amniotic fluid and meconium of mice whose mothers received food containing this species of bacteria. The marked bacteria were absent in mice from the control group [7]. Contact with microbes in the intrauterine environment could hold significance in the development of the immune system. The discovery of a potential vertical bacteria transfer has raised the interest in the maternal intestinal flora during pregnancy. Probiotic supplementation during pregnancy modulates the development of the immune system according to one of the studies [8]. Maternal probiotic supplementation substantially modulated the immune gene expression profile (TLR-related genes) both in the placenta and in the fetal gut. Final conclusions cannot be drawn until there is more research regarding this subject. The prenatal antibiotic exposure and its intensity could affect the composition of gut microbiota in preterm infants [9].

\section{Childbirth}

The delivery type and gestational age bear an impact on the microbiome composition in early life [10]. The vaginal microflora changes during pregnancy, its diversity diminishes and Lactobacillus species (L. iners, L. crispatus, L. jensenii,
L. johnsonii) emerges as dominant [11]. Newborns delivered by natural labour are primarily colonized by bacteria from maternal reproductive tract, belonging to the Lactobacillus, Bifidobacterium species and most likely intestinal Enterobacteriaceae species [12]. Contact with the mother's skin after birth enables the transfer of Staphylococci. Newborns delivered by cesarean section do not have direct contact with the maternal microbiota, instead, they're colonized by microflora characteristic for the skin and the hospital environment [13]. This is reflected in the intestinal microbiome composition of these newborns. Staphylococcus, Corynebacterium and Propionibacterium species become the dominant genera, with a considerably lower share of Bifidobacterium and Bacteroides species [14]. Differences in the intestinal microbiome of newborns delivered by cesarean section can be visible up to seven years after birth. Children born prematurely or with low birth weight will most likely exhibit a different microbiome composition [15]. It may be caused by a higher percentage of delivery by cesarean section in these cases, as well as more common perinatal antibiotic therapy. Newborns in these groups more often require prolonged hospitalization in a neonatal intensive care unit (NICU) and in many cases develop a characteristic for NICU non physiological flora [16]. Dominguez-Bello et al. investigated the possibility of restoration the physiological microflora of the newborn. One hour before the planned cesarean section, sterile gauze was placed in the mother's vagina, in order to wipe the mouth, face and the rest of the body of the newborn after delivery. It has been shown that microbiome in the anal swab of children that have undergone the aforementioned procedure held more similarities to the microbiome of newborns delivered naturally, than those from the control group [17]. This procedure, despite the potential benefits following a more physiological composition of the microbiome, is currently not recommended due to the need for closer examination of potential risks for the newborn ensuing from the procedure [18]. Concerns relate in particular to the exposure to pathogenic microorganisms, such as HSV, group B Streptococci, Chlamydia trachomatis, and Neisseria gonorrhoeae.

\section{Early years of life}

In the first months of life, the infant receives all nutrients via breast milk or a suitable formula. Breast milk facilitates the colonization and maturation of newborn's microbiota after birth. It contain sacteria [19, 20], the count and composition of which vary with the duration of the lactation, as well as the mother's age and health. Furthermore, the microbiome composition of the breast milk may change depending on the mother's caloric intake, her BMI, the delivery method and the sex of the child [21]. Bacteria present in breast milk belong primarily to Staphylococcus, Streptococcus, Serratia, 
Pseudomonas, Corynebacterium genera [22] as well as Bifidobacterium and Lactobacillus. The intestinal microbiome of infants fed naturally is dominated by Bifidobacterium and Bacteroides, which is most likely due to the presence of human milk oligosaccharides (HMO) in the milk, acting as prebiotics stimulating the growth of aforementioned bacteria. Formula-fed infants present a different intestinal microflora, dominated by Staphylococcus, anaerobic Streptococcus and Clostridium species. The introduction of solid foods is associated with a gradual transformation of the microbiome into the one observed in adults, which lasts until the age of three years [23]. The introduction of solid foods leads to a rapid increase in the number of bacteria from Enterobacteriaceae and Enterococcus species, with simultaneous further colonization by Bacteroides, Clostridium and anaerobic Streptococcus species. The type of consumed food and also have a significant impact on the intestinal microbiome [24]. Research on the development of the microbiome in the first three key years of life, especially on the intestinal microflora is crucial, due to its potential effect on individual development, along with the risk of certain diseases.

\section{Microbiome's impact on health}

Conclusions from numerous researches on the human microbiome show a correlation between the disturbances in the host's microbiome and an increased risk of certain diseases [25]. The presented correlation applies primarily to gastrointestinal, dermatological, neurodegenerative, neuropsychiatric, neuroimmune diseases, obesity and type 1 diabetes.

\section{Atopy}

Allergic diseases can be described as an early manifestation of the immune system dysregulation. Microbes residing in the infant's body have a significant role in shaping the immune system of the child. Exposure to environmental factors such as maternal infections, diet, bacterial colonization, toxic compounds may directly or indirectly influence the development of the immune system, both in utero and postnatal life. Exposure to certain microorganisms in early childhood reduces the risk of certain diseases such as inflammatory bowel disease (IBD) or asthma. However, no correlation has been shown between the place of birth and the predisposition to inhalation allergy [26] A study on germ-free mice has proven that exposure to commensal microbes in the early years of development is a protective factor against the development of these diseases [27]. Caesarean delivery has been shown to be associated with a higher risk of developing asthma in children, as children delivered via cesarean section in a hospital displayed a significantly higher percentage of $C$. difficile colonization. This correlation concerned children with a familial occurrence of atopy. Furthermore, a relation has been found for these children between cesarean delivery and a higher risk of food allergy and eczema. Another study has displayed a $40 \%$ occurrence reduction of atopic dermatitis in children of mothers, who received probiotics during pregnancy, compared to the control group [28]. Moreover, children delivered by cesarean section display a significantly lower level of Th1 response in the first two years of life, which is associated with an increased risk of developing allergic diseases.

\section{Gastrointestinal tract}

Recently, the study of the influence of microbes residing in the human digestive tract on the development of inflammatory diseases in strongly emphasized. Study of 447 children with Crohn's disease has shown a smaller variety of microbiome, an increased number of Enterobacteriaceae, Pasteurellaceae, Fusobacteriaceae, Neisseriaceae, Veillonellaceae and Gemellaceae, as well as a decreased percentage of Bifidobacteriaceae, Erysipelotrichaceae, Clostridiales and Bacteroidales, compared to the control group [29]. Brown et al. have shown in their earlier cohort study on stool samples from Finnish children taxonomical differences in the intestinal microflora composition between the children affected by autoimmune diseases and healthy ones. Further metagenomic analysis of the intestinal microbiome suggested a functional model of the development of autoimmunity in type 1 diabetes. The microbiome of healthy children turned out to be significantly more functionally diverse compared to the autoimmune microbiome [30]. Latest analyses suggest a possibility of preventing the development of type 1 diabetes by modulating the intestinal microbiota. Experiments on rats treated with Freund adjuvant and Lactobacillus strains have shown a delay or a decrease in diabetes occurrence [31].

\section{Neurodevelopment}

The composition of the intestinal microbiome is connected to the development of the neuronal connectivity system. Changes in its contents in the prenatal and postnatal period may have an influence on neurological development. It has been hypothesized that this phenomenon takes place through the microbiome-gut-brain axis, involving, among others, neurotrophic factors, susceptible to epigenetic regulation [32,33]. Extensive communication between the digestive tract and the brain through the gut-brain axis is present since the very early stages of the organism's development. The mechanism of the communication between the microbiota and the brain is not yet fully understood, however, it is postulated that they are affected by immunological, endocrine, metabolic and neuronal processes [34]. Mice deprived of the microbial exposition show underdevelopment of both innate and adaptive immune system. Recently 
scientists' attention is drawn to the role of the microbiome in neuropsychiatry [35]. It has been noticed that the intestinal bacteria affect behaviour and cognitive processes. Currently, the amount of data indicating an impact of the intestinal microbiota on the brain and behaviour keeps increasing [36]. Since it has been proven that the intestinal microbiome may influence the central nervous system, it has been proposed to include the microbiome as a port of this axis, calling it the microbiota-gut-brain axis or the microbiome-gut-brain axis (MGB). The intestinal microbiome is capable of affecting the host's gene expression, which is crucial if genes responsible for the functioning of the central nervous system are taken into account, probably through modulation of the hypothalamic-pituitary-adrenal axis, immune system, neurotransmitter and growth factor pathways, possibly also the limbic system, especially the amygdala and hypothalamus. Host microbiota is the main source of microbial antigens for the host, which are an important modulating factor in the development of the immune system, through promoting the formation of lymphoid tissue in the intestines. Furthermore, the intestines form an anatomical barrier, separating the visceral systems from local bacteria. Gut dysbiosis is associated with an increase of the immune cells' activity, cytokine production among others, which leads to an impairment of the intestinal barrier and subsequent chronic inflammation. The presence of neuropsychiatric diseases is often connected to disorders of the gastrointestinal tract, which are associated with the intestinal dysbiosis. Stress in early childhood, such as separation from the mother in the neonatal period, is associated with an intestinal dysfunction [37]. A specific microbiome has also been observed in depression, comprising of an increased count of Bacteroidales and decrease of Lachnospiraceae, as well as an increase in the population of Alistipes and Enterobacteriaceae together with a decreased Faecalibacterium percentage [38]. Moreover, a protective effect of the last group on the development of neuropsychiatric diseases, including depression, has been noted. The presented data indicate a significant association between the intestinal microflora and the central nervous system through communication at the MGB axis level. This association may affect the pathophysiology of depression, developing since the prenatal period until adulthood, on the foundation of early stress factors [39]. Extensive communication between the digestive tract and the brain through the gut-brain axis is present since the very early stages of the organism's development and could be a potent target for the treatment of neuropsychiatric disorders, including depression, due to its bi-directionality [40].

\section{SUMMARY}

Previous research on the subject of the human microbiome shows that the course of pregnancy, delivery type, as well as the type of feeding of babies, influence the development of the human microbiome at an early stage of life. This correlation causes a higher incidence of certain diseases and disorders in those children who show abnormalities in the composition of their microbiome, mainly intestinal. Many studies still need to be confirmed or extended in order to be able to draw far-reaching conclusions.

\section{REFERENCES}

1. Tanaka M, Nakayama J. Development of the gut microbiota in infancy and its impact on health in later life. Allergol Int. 2017; 66(4): 515-522, doi: 10.1016/j.alit.2017.07.010, indexed in Pubmed: 28826938.

2. Janda JM, Abbott SL. $16 \mathrm{~S}$ rRNA gene sequencing for bacterial identification in the diagnostic laboratory: pluses, perils, and pitfalls. J Clin Microbiol. 2007; 45(9): 2761-2764, doi: 10.1128/JCM.01228-07, indexed in Pubmed: 17626177.

3. Schubert S, Kostrzewa M. MALDI-TOF MS in the Microbiology Laboratory: Current Trends. Curr Issues Mol Biol. 2017; 23: 17-20, doi: 10.21775/cimb.023.017, indexed in Pubmed: 28504240.

4. Rehbinder EM, Lødrup Carlsen KC, Staff AC, et al. Is amniotic fluid of women with uncomplicated term pregnancies free of bacteria? Am J Obstet Gynecol. 2018; 219(3): 289.e1-289.e12, doi: 10.1016/j. ajog.2018.05.028, indexed in Pubmed: 29852156.

5. Aagaard K, Ma J, Antony KM, et al. The placenta harbors a unique microbiome. Sci Transl Med. 2014; 6(237): 237ra65, doi: 10.1126/scitranslmed.3008599, indexed in Pubmed: 24848255.

6. Rautava S, Collado MC, Salminen S, et al. Probiotics modulate host-microbe interaction in the placenta and fetal gut: a randomized, double-blind, placebo-controlled trial. Neonatology. 2012; 102(3): 178-184, doi: 10.1159/000339182, indexed in Pubmed: 22776980.

7. Jiménez E, Marín ML, Martín R, et al. Is meconium from healthy newborns actually sterile? Res Microbiol. 2008; 159(3): 187-193, doi: 10.1016/j. resmic.2007.12.007, indexed in Pubmed: 18281199.

8. Rautava S, Collado MC, Salminen S, et al. Probiotics modulate host-microbe interaction in the placenta and fetal gut: a randomized, double-blind, placebo-controlled trial. Neonatology. 2012; 102(3): 178-184, doi: 10.1159/000339182, indexed in Pubmed: 22776980.

9. Zou ZH, Liu D, Li HD, et al. Prenatal and postnatal antibiotic exposure influences the gut microbiota of preterm infants in neonatal intensive care units. Ann Clin Microbiol Antimicrob. 2018; 17(1): 9, doi: 10.1186/s12941018-0264-y, indexed in Pubmed: 29554907.

10. Hill CJ, Lynch DB, Murphy K, et al. Evolution of gut microbiota composition from birth to 24 weeks in the INFANTMET Cohort. Microbiome. 2017; 5(1): 4, doi: 10.1186/s40168-016-0213-y, indexed in Pubmed: 28095889.

11. Aagaard K, Riehle K, Ma J, et al. A metagenomic approach to characterization of the vaginal microbiome signature in pregnancy. PLoS One. 2012; 7(6): e36466, doi: 10.1371/journal.pone.0036466, indexed in Pubmed: 22719832.

12. de Muinck EJ, Oien T, Storrø O, et al. Diversity, transmission and persistence of Escherichia coli in a cohort of mothers and their infants. Environ Microbiol Rep. 2011;3(3):352-359, doi: 10.1111/j.1758-2229.2010.0023 1.x, indexed in Pubmed: 23761281.

13. Dominguez-Bello MG, Costello EK, Contreras M, et al. Delivery mode shapes the acquisition and structure of the initial microbiota across multiple body habitats in newborns. Proc Natl Acad Sci U S A. 2010; 107(26): 11971-11975, doi: 10.1073/pnas.1002601107, indexed in Pubmed: 20566857.

14. Azad MB, Konya $\mathrm{T}$, Maughan $\mathrm{H}$, et al. CHILD Study Investigators. Gut microbiota of healthy Canadian infants: profiles by mode of delivery and infant diet at 4 months. CMAJ. 2013; 185(5): 385-394, doi: 10.1503/cmaj.121189, indexed in Pubmed: 23401405.

15. Forsgren $M$, Isolauri $E$, Salminen $S$, et al. Late preterm birth has direct and indirect effects on infant gut microbiota development during the first six months of life. Acta Paediatr. 2017; 106(7): 1103-1109, doi: 10.1111/apa.13837, indexed in Pubmed: 28316118.

16. Groer MW, Luciano AA, Dishaw LJ, et al. Development of the preterm infant gut microbiome: a research priority. Microbiome. 2014; 2: 38, doi: 10.1186/2049-2618-2-38, indexed in Pubmed: 25332768.

17. Dominguez-Bello MG, De Jesus-Laboy KM, Shen N, et al. Partial restoration of the microbiota of cesarean-born infants via vaginal microbial transfer. Nat Med. 2016; 22(3): 250-253, doi: 10.1038/nm.4039, indexed in Pubmed: 26828196. 
18. American College of Obstetricians and Gynecologists' Committee on Obstetric Practice in collaboration with Kurt R. Wharton, and Meredith L. Birsner. Vaginal Seeding. ACOG COMMITTEE OPINION. 2017; 725.

19. Jost $T$, Lacroix $C$, Braegger $C P$, et al. Vertical mother-neonate transfer of maternal gut bacteria via breastfeeding. Environ Microbiol. 2014; 16(9): 2891-2904, doi: 10.1111/1462-2920.12238, indexed in Pubmed: 24033881.

20. Jeurink PV, van Bergenhenegouwen J, Jiménez $E$, et al. Human milk: a source of more life than we imagine. Benef Microbes. 2013;4(1): 17-30, doi: 10.3920/BM2012.0040, indexed in Pubmed: 23271066.

21. Williams JE, Carrothers JM, Lackey KA, et al. Human Milk Microbial Community Structure Is Relatively Stable and Related to Variations in Macronutrient and Micronutrient Intakes in Healthy Lactating Women. J Nutr. 2017; 147(9): 1739-1748, doi: 10.3945/jn.117.248864, indexed in Pubmed: 28724659.

22. Hunt KM, Foster JA, Forney $\amalg$, et al. Characterization of the diversity and temporal stability of bacterial communities in human milk. PLoS One. 2011;6(6): e21313, doi: 10.1371/journal.pone.0021313, indexed in Pubmed: 21695057.

23. Yatsunenko T, Rey FE, Manary MJ, et al. Human gut microbiome viewed across age and geography. Nature. 2012; 486(7402): 222-227, doi: 10.1038/nature11053, indexed in Pubmed: 22699611.

24. O'Grady J, O'Connor EM, Shanahan F. Review article: dietary fibre in the era of microbiome science. Aliment Pharmacol Ther. 2019; 49(5): 506-515, doi: 10.1111/apt.15129, indexed in Pubmed: 30746776.

25. Avershina E, Cabrera Rubio R, Lundgård K, et al. Effect of probiotics in prevention of atopic dermatitis is dependent on the intrinsic microbiota at early infancy. J Allergy Clin Immunol. 2017; 139(4): 1399-1402.e8, doi: 10.1016/j.jaci.2016.09.056, indexed in Pubmed: 27931973.

26. van Nimwegen FA, Penders J, Stobberingh EE, et al. Mode and place of delivery, gastrointestinal microbiota, and their influence on asthma and atopy. J Allergy Clin Immunol. 2011; 128(5): 948-955.e1, doi: 10.1016/j. jaci.2011.07.027, indexed in Pubmed: 21872915.

27. Rogers GB, Keating DJ, Young RL, et al. From gut dysbiosis to altered brain function and mental illness: mechanisms and pathways. Mol Psychiatry. 2016; 21(6): 738-748, doi: 10.1038/mp.2016.50, indexed in Pubmed: 27090305.

28. Avershina $E$, Cabrera Rubio R, Lundgård K, et al. Effect of probiotics in prevention of atopic dermatitis is dependent on the intrinsic microbiota at early infancy. J Allergy Clin Immunol. 2017; 139(4): 1399-1402.e8, doi: 10.1016/j.jaci.2016.09.056, indexed in Pubmed: 27931973.

29. Tognini P. Gut Microbiota: A Potential Regulator of Neurodevelopment. Front Cell Neurosci. 2017; 11:25, doi: 10.3389/fncel.2017.00025, indexed in Pubmed: 28223922.
30. Jakobsson HE, Abrahamsson TR, Jenmalm MC, et al. Decreased gut microbiota diversity, delayed Bacteroidetes colonisation and reduced Th1 responses in infants delivered by caesarean section. Gut. 2014; 63(4): 559-566, doi: 10.1136/gutjnl-2012-303249, indexed in Pubmed: 23926244.

31. Lau K, Benitez P, Ardissone A, et al. Inhibition of type 1 diabetes correlated to a Lactobacillus johnsonii N6.2-mediated Th17 bias. J Immunol. 2011; 186(6): 3538-3546, doi: 10.4049/jimmunol.1001864, indexed in Pubmed: 21317395.

32. Rea K, Dinan TG, Cryan JF. The microbiome: A key regulator of stress and neuroinflammation. Neurobiol Stress. 2016; 4: 23-33, doi: 10.1016/j. ynstr.2016.03.001, indexed in Pubmed: 27981187.

33. Park $\mathrm{H}$, Poo Mm. Neurotrophin regulation of neural circuit development and function. Nat Rev Neurosci. 2013; 14(1): 7-23, doi: 10.1038/nrn3379, indexed in Pubmed: 23254191.

34. Collado MC, Rautava S, Aakko J, et al. Human gut colonisation may be initiated in utero by distinct microbial communities in the placenta and amniotic fluid. Sci Rep. 2016; 6: 23129, doi: 10.1038/srep23129, indexed in Pubmed: 27001291.

35. Brown CT, Davis-Richardson AG, Giongo A, et al. Gut microbiome metagenomics analysis suggests a functional model for the development of autoimmunity for type 1 diabetes. PLoS One. 2011; 6(10): e25792, doi: 10.1371/journal.pone.0025792, indexed in Pubmed: 22043294.

36. Bäckhed F, Roswall J, Peng Y, et al. Dynamics and Stabilization of the Human Gut Microbiome during the First Year of Life. Cell Host Microbe. 2015; 17(5): 690-703, doi: 10.1016/j.chom.2015.04.004, indexed in Pubmed: 25974306.

37. Lennon EM, Maharshak N, Elloumi $\mathrm{H}$, et al. Early life stress triggers persistent colonic barrier dysfunction and exacerbates colitis in adult IL-10-/- mice. Inflamm Bowel Dis. 2013; 19(4): 712-719, doi: 10.1097/MIB.0b013e3182802a4e, indexed in Pubmed: 23446335.

38. Jiang $\mathrm{H}$, Ling $Z$, Zhang $\mathrm{Y}$, et al. Altered fecal microbiota composition in patients with major depressive disorder. Brain Behav Immun. 2015; 48: 186-194, doi: 10.1016/j.bbi.2015.03.016, indexed in Pubmed: 25882912.

39. Foster JA, McVey Neufeld KA. Gut-brain axis: how the microbiome influences anxiety and depression. Trends Neurosci. 2013; 36(5): 305-312, doi: 10.1016/j.tins.2013.01.005, indexed in Pubmed: 23384445.

40. Lima-Ojeda J, Rupprecht R, Baghai T. "I Am I and My Bacterial Circumstances": Linking Gut Microbiome, Neurodevelopment, and Depression. Frontiers in Psychiatry. 2017; 8, doi: 10.3389/fpsyt.2017.00153. 